\title{
A Low-Cost Energy Management System That Compares Power Consumption of Electronic Home Appliances
}

\author{
${ }^{1}$ Dushyanth K, ${ }^{2} \mathrm{G}$ Leenendra Chowdary, ${ }^{3} \mathrm{~N}$ V G Prasad \\ ${ }^{1}$ M.Tech, Student ${ }^{2}$ Asst. Professor ${ }^{3}$ Asso. Professor, ${ }^{3}$ Head of the Department, \\ ${ }^{1,2,3}$ Sasi Institute of Technology and Engineering, Tadepalligudem. INDIA.
}

\begin{abstract}
The main objective of the proposed system is to compare the power consumption of any electrical appliances both domestic and industrial. Here we propose a system that is cost effective by minimal system integration to achieve the goal. A typical HEMS just shows the energy consumption of the hole home and home appliances. Users cannot figure-out how efficient a home appliance is to that of others. So it is necessary to compare the energy usage of the home appliances to that of the same kinds of home appliances. In this paper we propose a low-cost EMS with minimal system integration based on energy comparison
\end{abstract}

Keywords-EMM, HEMM, HEMS.

\section{INTRODUCTION}

In the present days Energy saving has become an important consideration and a research issue. The Home Energy Management System (HEMS) technology has helped this concern to effectively monitor the energy usage of the entire home. And we purposed to contribute this work inorder to analyse the energy usage and the performance of a particular or a group of similar kind of home appliances by comparing their energy consumptions separately or as a group. The system is also cost-effective and of minimal size in its architecture with limited system integration.

\section{PROPOSED SYSTEM}

The proposed system is built around the ARM7 TDMI Microcontroller LPC2148 to which the Microchip's Energy metering IC ADE7757 is interfaced and a Zigbee module for low-cost wireless communication to adapt communication with the monitoring station. The home appliances are connected to the mains via the Home Energy Measuring Module (HEMM) which has a function of energy measuring of home appliances and also has the capability of Zigbee communication.

The Zigbee network is well known as a low power communication method. The Energy monitoring module in the monitoring station identifies the home appliances by their corresponding Energy measuring module number. The energy measuring module measures the real-time active power consumption. The Monitoring module collects the details of energy consumption from each measuring module via Zigbee communication sends it to the PC. The energy consumption details can be viewed in the PC's Hyper-terminal Communication window. The architecture of the entire network is shown in Fig 1.

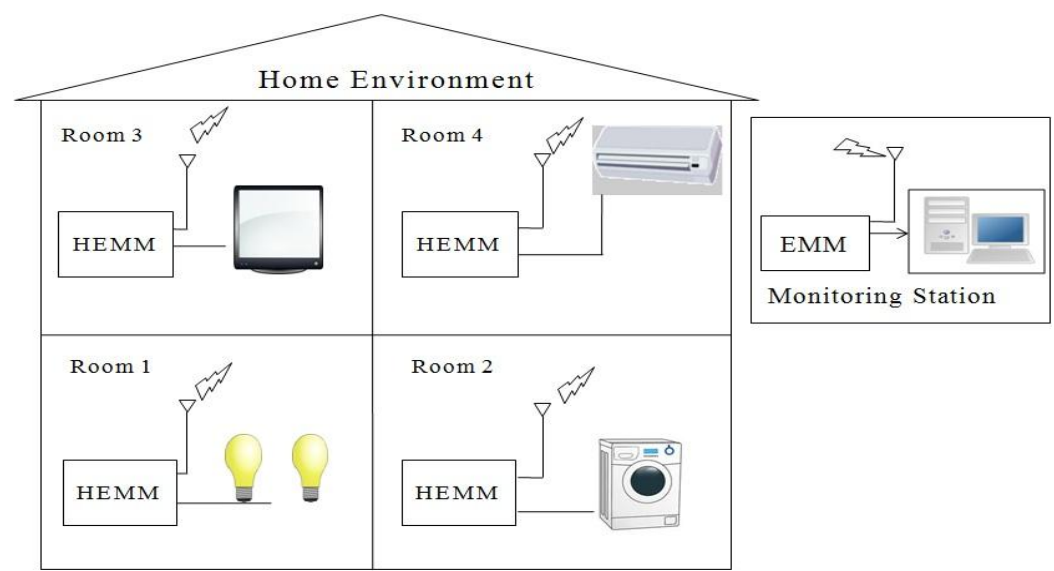

figure1. Architecture of the proposed system 


\section{SYSTEM HARDWARE}

The System Hardware consists of Power supply module, Energy meter module, Controller, Wireless communication module, Relay control, and LCD module. The power supply module gives 5Volts DC and 3.3V DC as the system requires multiple operating voltages, the LPC2148 operates using $3.3 \mathrm{v}$ and the remaining modules uses $5 \mathrm{~V}$.

The energy meter is constructed on the ADE7757 which is an energy metering IC with integrated Oscillator thus eliminating the cost of external crystal or resonator, thus reducing the overall cost. The chip directly interfaces with the shunt resistor and operates only with AC input. The only analog part used in it is the $\sum-\triangle$ ADCs and reference circuits. All other signal processing is carried out in the digital domain. Thus provides superior stability and accuracy over time and extreme environmental conditions.

The IC includes a power supply monitoring circuit on the Vdd supply pin. The ADE7757 will remain in-active until the supply voltage on Vdd reaches approximately 4Volts. Due to this, proper device operation is achieved at power-up and power-down modes. High degree of immunity to false triggering from noisy supplies is attained due to built-in hysteresis and filtering operation in power supply monitor of the ADE7757. One more interesting feature in the ADE7757 IC is that it detects when the current and voltage channels have a phase shift greater than 90degree. This mechanism can detect wrong connection of the meter or generation of negative power. The construction details are shown in the block diagram representation in Fig 2.

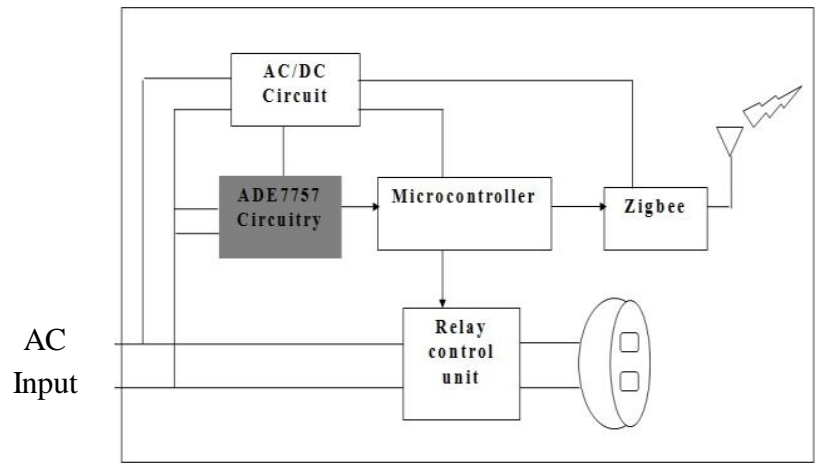

figure2. Block diagram of Energy Measuring Module (HEMM)

Choosing an appropriate Wireless Communication System is one of the main tasks. Zigbee is a recently developed two-way wireless communication protocol designed to meet very low power consumption and low cost. The higher protocol layers are being defined by the Zigbee Alliance group while interests in the lower layers of the stack are being defined by the IEEE 802.15 working group 4 (802.15.4) which is aimed at achieving data throughput of $250 \mathrm{kbps}$ in the $2.4 \mathrm{GHz}$ band and Signees can be implemented in Network layer and Application layer. Zigbee has been developed to meet the growing demand for capable wireless networking between numerous low-power devices. In order to solve these problems, we can consider using of Zigbee Wireless Communication system which is popularly used in the world, to transmit power data. Here Zigbee based wireless communication sub-system is responsible for receiving and transferring data. Zigbee wireless open standard technology is being selected around the world as the energy management and efficiency technology of choice in terms of reliability and timing. And the system uses Zigbee wireless communication module (XBee 2.5). It is used to transfer the data of the user meter from LPC2148 controller to Monitoring Module by Zigbee wireless module.

Relay control unit is used to control the functioning of the home appliances. The relay is driven by the LPC3148 controller.

The Energy consumption data can be viewed in the LCD interfaced to the LPC2148 controller at the Energy Measuring module. The controller is a 32-bit ARM7 controller LPC2148 and the data can be transferred to the Monitoring Module through communication module.

The controller LPC2148 is based on a 16-bit/32-bit ARM7TDMI-S CPU with real-time emulation and embedded trace support, that combine microcontroller with embedded high-speed flash memory of $512 \mathrm{kB}$. A 128-bit wide memory interface and unique accelerator architecture enable 32-bit code execution at the maximum clock rate. Due to their tiny size and low power consumption, LPC2148 are ideal for applications where miniaturization is a key requirement and this controller best fits the proposed system since the system would be like a smart phone with good user interface when modified into a full consumer product. And also the controller has serial communication interfaces from a USB 2.0, multiple UARTs, SPI and SSP to I2C bus where some of them were useful in system integration with its peripheral devices just like the EEPROM which uses I2C bus to store data. 


\section{IMPLEMENTATION DETAILS}

The proposed system is built around Three ARM7 LPC 2148 based boards. The LPC2148 has an onchip timer, interrupts and UART and IO ports for interfacing energy meter. The code is written in Embedded C programming used for programming the ARM. When the system is turned on, the energy meter used provides output pulses indicating the energy burnt. It provides pulses for every one unit of energy consumed by the appliance. The pulses are given to the ARM controller which counts the pulses and increments the internal counter. The counter counts the number of units and increments the units count in the controller. The data regarding the consumption is saved into EEPROM not to lose the data if any error occurs while wireless transmission or power failure. A timer is initialized to give an interrupt for a preset time by the user which initializes serial communication and transmits the units consumed via Zigbee wireless communication to the monitoring environment which is Energy Monitoring Module (EMM). At the monitoring environment the data is received by the Zigbee module and sent to the controller and serially transmitted to the PCs' Hyper-terminal.

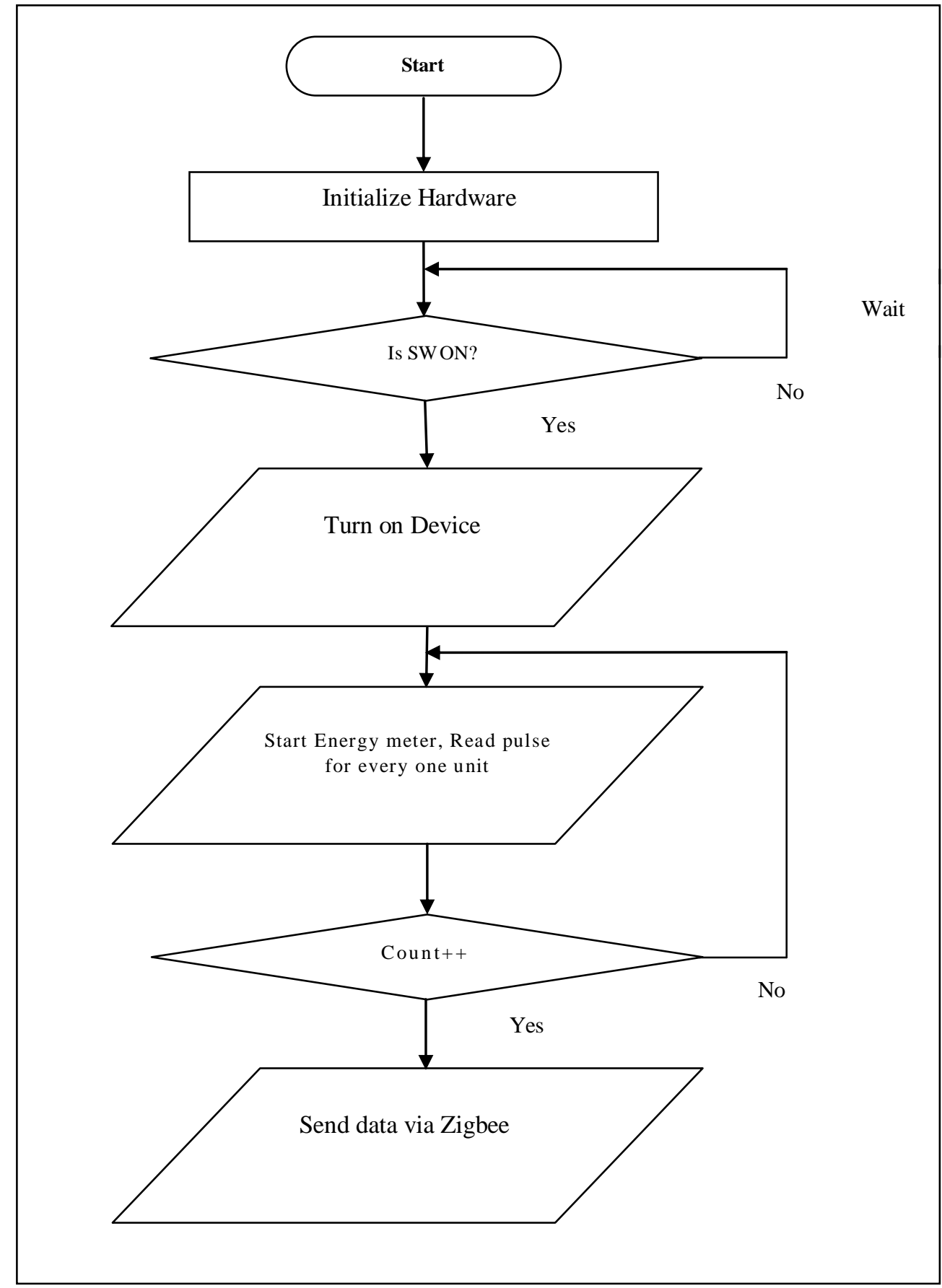

figure 3 shows the code implementation sequence of a HEMM 
The user can view the data regarding the units consumed and can compare the energy consumption of the home appliances likewise. This would help the user to make choices regarding the usage of particular appliance in the home environment. The flowchart in figure 3 shows the code implementation sequence.

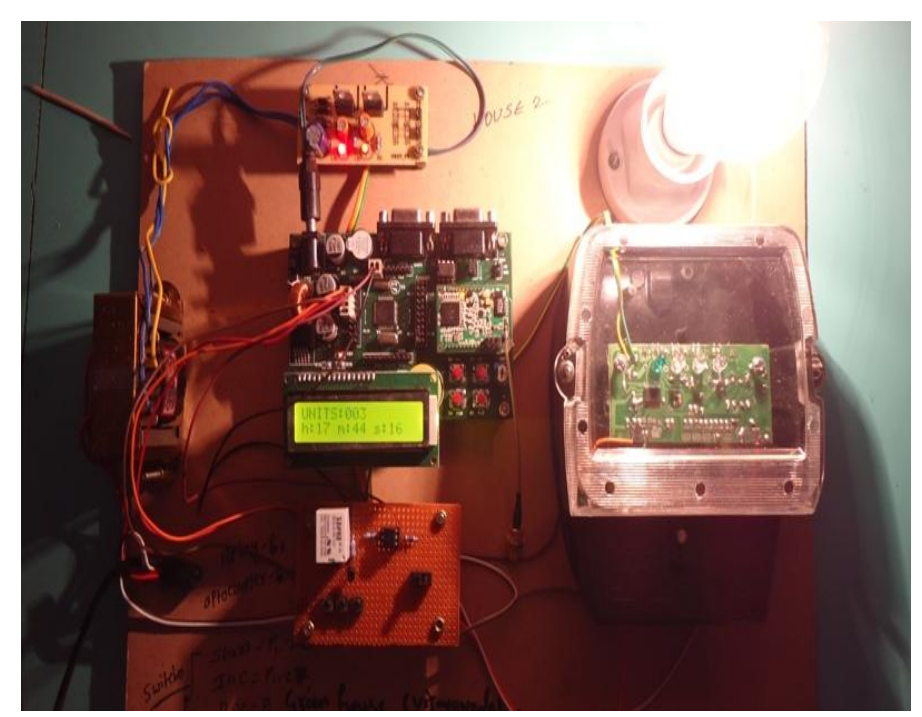

figure 4. Prototype of HEMM

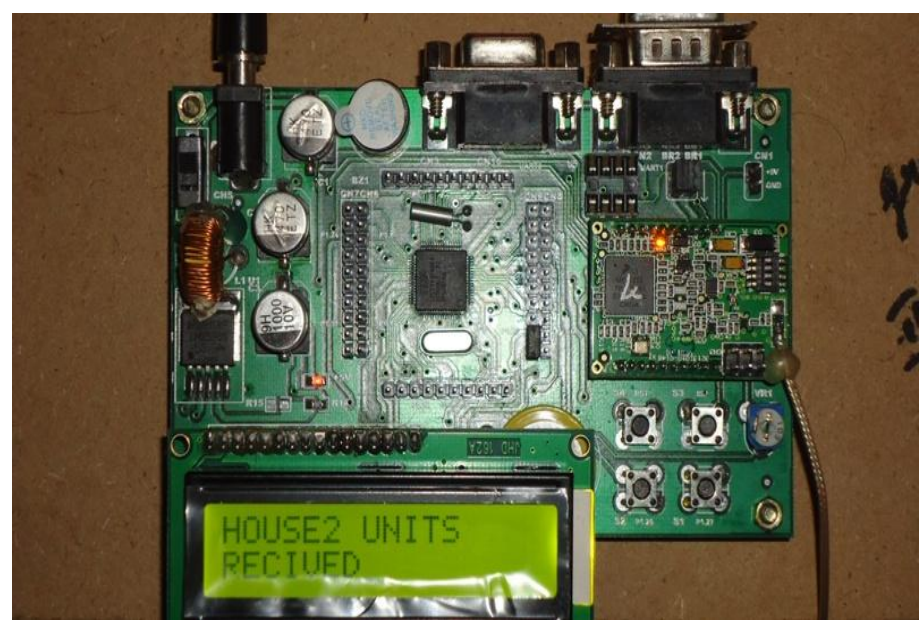

figure 5. Prototype of EMM

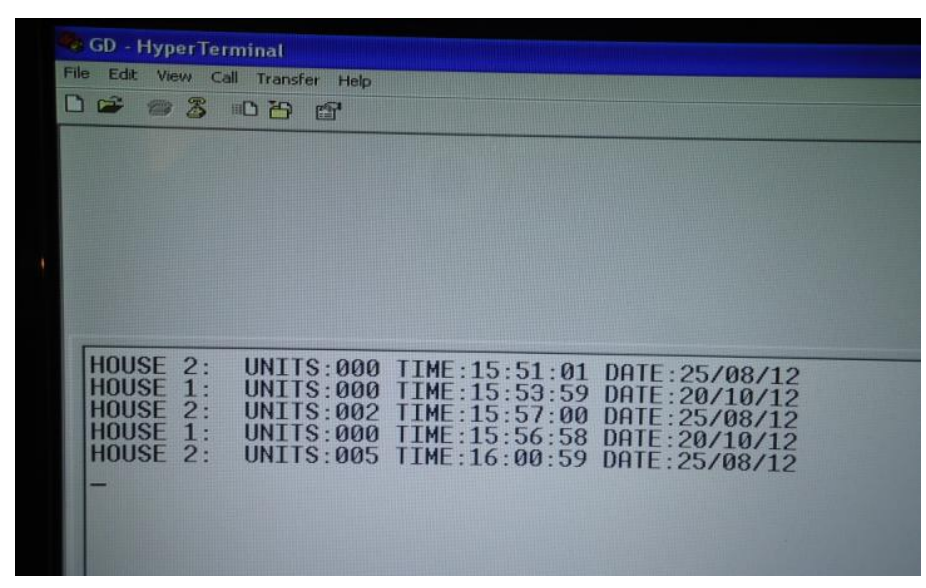

figure 6. Output result of the system 


\section{RESULTS}

The entire setup is made in to prototype of three modules HEMM 1 HEMM 2 and EMM and the test environment is created using two bulbs of same wattage and then after with different wattages to each of the HEMMs and the data is obtained at the EMM which is the Monitoring environment and the results can be seen in the PCs HyperTerminal window. The figures 4 and 5 show the actual pictures of the prototype setup and figure 6 shows the system results.

\section{CONCLUSION}

In the present work the system is designed to monitor the real power with the help of ADE7757 IC which also reduced additional hardware and eased software development and ultimately reduced cost. The use of Zigbee wireless communication makes the system with advanced feature. The effective use of serial communication protocol helps the user to view the energy consumption data without data loss and that is achieved by the use of EEPROM. Thus the system is minimal in system integration, cost-effective and advance in technology.

\section{REFERENCES}

[1] Zigbee Home Automation: The New Global Standard For Home Automation.

[2] Michal Varchola, Miloš Drutarovský, Zigbee Based Home Automation Wireless Sensor Network, Acta Electrotechnica Et Informatica No. 4, Vol. 7, 2007.

[3] Zigbee Alliance, Zigbee Specification, December 1, 2006, [Online].

[4] Zbee802.15.4_At_Command_Specification.

[5] Yang Zhen Microchip Technology Inc. Micro Chip Application Note An1332 "Current Sensing Circuit Concepts And Fundamentals".

[6] Arm7tdmi-S Revision: R4p3, “Technical Reference Manual”. Web Address Http://Www.Arm.Com.

[7] “The Insider's Guide To The Philips Arm7-Based Microcontrollers" An Engineer's Introduction To The Lpc2100 Series, Trevor Martin Bsc. (Hons.) Ceng. Miee

[8] Um10139 Lpc214x User Manual, Rev. 3 - 4 October 2010.

[9] Ade7757 Data Sheet Of Analog Devices Energy Metering Ic

[10] Application Note An559, “A Low-Cost Watt-Hour Energy Meter Based On Ade7755

\section{AUTHORS}

Dushyanth Kundanati received his B.Tech in 2007, and pursuing M.Tech in VLSI \&ES at SASI Institute of Technology and Engineering, Tadepalligudem. India. He has gained good working experience on 8-bit controllers for the past few years. His areas of interests include Microcontrollers, Home Automation system design and Wireless Communications.

G Leenendra Chowdary received his M.Tech in VLSI \& ES and has over six years of experience in teaching various subjects. Presently he is an Assistant Professor in the Department of Electronics and Communication Engineering at SASI Institute of Technology and Engineering, Tadepalligudem. India. His areas of interest include VLSI Design Technology, Advanced Microcontrollers and Sensor networks.

N V G Prasad is an Associate Professor and is the Head of the Department for the Department of Electronics and Communication Engineering at SASI Institute of Technology and Engineering, Tadepalligudem. India. He has over ten years of experience in teaching various subjects and his areas of interests include Digital Image Processing, Digital Signal Processing, Advanced Microcontrollers and Advanced Communication Systems. 